\title{
Balsalazide led to greater remission rates and tolerance than mesalamine in acute ulcerative colitis
}

\author{
Green $\mathcal{F R}$, Lobo AF, Holdsworth CD, et al, and the ABACUS Investigator Group. Balsalazide is more effective and \\ better tolerated than mesalamine in the treatment of acute ulcerative colitis. Gastroenterology 1998 \\ fan; 114:15-22.
}

\section{Question}

In patients with acute ulcerative colitis, does balsalazide lead to greater remission rates and tolerance than mesalamine?

Design

12 week randomised, double blind, controlled trial.

\section{Setting}

19 clinical centres in the UK.

\section{Patients}

101 patients who were $18-80$ years of age and had symptomatic (moderate or severe) grade 2 to 4 ulcerative colitis that extended $\geqslant 12 \mathrm{~cm}$ beyond the anal margin. Exclusion criteria were Crohn's disease, idiopathic proctitis, noninflammatory bowel diseases, stool parasites, toxins, pathogens, use of steroids within the previous month, use of immunosuppressive agents in the previous 3 months, daily use of a rectal steroid to maintain remission, or use of mesalamine releasing compound releasing $\geqslant 1.2 \mathrm{~g} /$ day in the previous 14 days. Follow up was $98 \%$ (mean age $41 \mathrm{y}$, $63 \%$ men).

\section{Intervention}

Patients were allocated to balsalazide (Penn Pharmaceuticals, Tredegard, Gwent, UK), $2.25 \mathrm{~g}$ three times daily $(n=50)$, or mesalamine, $0.8 \mathrm{~g}$ three times daily $(n=49)$, for 4,8 , or 12 weeks as necessary. Patients were given a rectal steroid foam (hydrocortisone acetate [Colifoam]) for relief medication.

\section{Main outcome measures}

Complete remission (none or mild symptoms, no use of rectal steroid foam, and grade 0 or 1 ulcerative colitis on sigmoidoscopy). Secondary outcome measures were symptom relief and adverse events.

\section{Main results}

By 12 weeks, more patients treated with balsalazide than with mesalamine had achieved complete remission $(62 \% v$ $37 \%, \mathrm{p}=0.02)$; this effect was evident by week 4 . More patients in the balsalazide group than in the mesalamine group had achieved symptomatic remission (none or mild symptoms) by weeks $2(64 \%$ v $43 \%, \mathrm{p}=0.04), 8(78 \% v$ $45 \%, \mathrm{p}<0.001)$, and $12(88 \%$ v 57\%, p<0.001). After 2 weeks of treatment, patients in the balsalazide group rated their symptoms as being less severe than did those in the mesalamine group $(\mathrm{p}=<0.001)$; this effect remained at weeks $4(\mathrm{p}<0.05), 8(\mathrm{p}=0.001)$, and $12(\mathrm{p}=0.003)$. The median time to complete relief of symptoms was shorter for patients in the balsalazide group than in the mesalamine group (10 $v 25$ days, $\mathrm{p}=0.004)$. Patients treated with balsalazide had more days without symptoms and use of relief medication than did those treated with mesalamine during the first 4 weeks of treatment (mean $24 \% v 14 \%$, $\mathrm{p}=0.008$ ). Fewer patients treated with balsalazide than with mesalamine reported adverse events (48\% $v 71 \%$, $\mathrm{p}=0.02)$. Fewer patients in the balsalazide group than in the mesalamine group did not complete the study because of treatment failure $(12 \% v 33 \%, \mathrm{p}=0.015)$.

\section{Conclusion}

In patients with acute ulcerative colitis, balsalazide led to greater remission rates and tolerance than did mesalamine.

Source of funding: none stated.

For correspondence: Dr $\mathcal{F}$ R Green, City General Hospital, Newcastle Road, Stoke-onTrent, ST4 6QG, England, UK. Fax +44 (0) 1782712052.

\section{Commentary}

Green et al enrolled 101 (99 evaluable) patients with moderate to severe symptoms of ulcerative colitis (UC) into a randomised, double blind, double dummy trial comparing $6.75 \mathrm{~g}$ balsalazide (Colazide) and $2.4 \mathrm{~g}$ mesalamine (Asacol) over 12 weeks. The primary endpoint was complete remission defined as absent or mild symptoms with either a normal sigmoidoscopy or a sigmoidoscopy demonstrating erythema and loss of vascular pattern. $62 \%$ of balsalazide versus $37 \%$ of mesalamine treated patients achieved remission by 12 weeks. In addition, balsalazide treated patients had a shorter median time to complete relief, a higher percentage of symptom-free days, less treatment failures, and fewer serious adverse effects. The authors concluded that balsalazide was "more effective...with more favourable side effect profile".

This study suggests the clinical superiority of balsalazide over mesalamine in the formulations tested. The context, methodology and interpretations must, however, be viewed in perspective. This trial compared equivalent amounts of 5-aminosalicylic acid (5-ASA) delivered from Colazide and Asacol at currently "approved" doses of Asacol (2.4 g/day in the USA). This is not an optimal dose; prior mesalamine studies have demonstrated a dose response up to $4.8 \mathrm{~g} /$ day. Furthermore, in prior comparative trials, balsalazide has been therapeutically similar to sulfasalazine. In one trial, for instance, $50 \%$ "remission" rates were shown in both groups. Similarly, in a second trial, treatment success was reported in $81 \%$ of patients treated with balsalazide compared with $61 \%$ with sulfasalazine; this was not a statistically significant difference. In addition, a prior trial of 154 patients with mild to moderate UC who received equal amounts of 5-ASA, $23 \%$ of patients receiving balsalazide versus $19 \%$ of patients on Asacol (NS) were in remission at the end of 8 weeks. Finally, a 4 week trial demonstrated no statistical difference between balsalazide and placebo for the endpoints of stool frequency, blood in stool, sigmoidoscopic improvement, Physician's Global Assessment, or overall assessment.

Thus, although the current trial suggests superiority of balsalazide, the results must be interpreted with caution. In larger meta-analyses, it has not been possible to distinguish efficacy advantages between oral 5-ASA delivery systems. Compared with sulfasalazine, all of the non-sulfa aminosalicylates are better tolerated. In comparing aminosalicylate formulations in clinical trials, it is essential to consider study details including sample sizes, study populations of disease extent and severity, doses, endpoints, and duration of treatment.

$S$ B HANAUER

University of Chicago Medical Centre, 5758 S Maryland MC 9028,

Chicago, IL 60637, USA

1 Anonymous. Colazide Monograph. UK: Biorex

2 Riley SA. What dose of 5-aminosalicylic acid (mesalazine) in ulcerative colitis? Gut 1998;42: 761-3. 